\title{
Mechanismen genetischer Epilepsien
}

\author{
Ulrike Hedrich, Snezana Maljevic und Holger Lerche
}

\section{Zusammenfassung}

Epilepsie, früher auch als Fallsucht bezeichnet, ist eine der häufigsten neurologischen Erkrankungen. Bereits zu Hippokrates' Zeiten (460 - 370 v. Chr.) wurde von der sogenannten „Heiligen Krankheit" berichtet (Fröscher 2004). Heute weiß man, dass es sich bei einem epileptischen Anfall um eine Folge synchroner Entladungen von Neuronengruppen im Gehirn handelt, die plötzlich und ohne vorhergehende erkennbare Ursache zu unwillkürlichen Befindens-, Verhaltens- oder Bewusstseinsstörungen führen. Solche Störungen können unterschiedlichste Ursachen und bei den betroffenen Patienten erhebliche Auswirkungen auf das Alltagsleben haben. Bis zu 50 \% aller Epilepsien sind dabei genetisch bedingt, insbesondere die idiopathischen Epilepsien, bei denen sich keine Hinweise auf eine äußere Ursache oder auf Veränderungen des Gehirns finden. Genetisch bedingte Funktionsstörungen von neuronalen Ionenkanälen spielen eine zentrale Rolle bei der Entstehung solcher Epilepsien. Diese Ionenkanäle steuern den Ionenfluss durch die Zellmembran von Nervenzellen und stellen die Basis für die Erregbarkeit dieser Nervenzellen dar. Deshalb wirken auch Medikamente, die zur Behandlung von Epilepsien eingesetzt werden, überwiegend auf Ionenkanäle. Dabei sind die bekannten Antiepileptika nur z.T. erfolgreich, da ein Drittel der Patienten eine Pharmakoresistenz aufweist und bei anderen viele Nebenwirkungen auftreten. Weiterhin ist die Behandlung ausschließlich symptomatisch und wirkt nicht auf die epileptogenen Mechanismen, die noch weitgehend unbekannt sind. In diesem Übersichtsartikel werden wir derzeitige Forschungsschwerpunkte genetisch bedingter Epilepsien und deren Pathomechanismen näher beleuchten sowie Therapiemöglichkeiten vorstellen.

\begin{abstract}
Mechanisms of genetic epilepsies.

Epilepsy is one of the most common neurological disorders. Already at the time of Hippocrates (460 - 370 BC) it was reported on as the "holy disease" (Fröscher 2004). Today it is known that an epileptic seizure is a consequence of synchronous discharges of neuronal populations in the brain, which abruptly and usually without an observable cause evoke involuntary behavioural dysfunction or impaired consciousness. Epilepsies can have various causes and lead to extensive implications for the everyday life of affected patients. Up to $50 \%$ of all epilepsies are caused by genetic defects, in particular the so-called idiopathic epilepsies which occur without easily observable structural alterations of the brain. Genetically caused dysfunctions of neuronal ion channels play a central role in the formation of such epilepsies. The ion channels control the ion flux over the cell membrane of neurons and thus present the basis for the excitability of these neurons. Therefore, medications used for epilepsy treatment affect predominantly ion channels. However, the common anticonvulsants have limited success, not only because one third of epilepsy patients exhibits pharmacoresistance, but also because of the secondary effects which can dramatically affect their quality of life. Furthermore, current therapeutic approaches are mainly symptomatic and do not act on the epileptogenic mechanisms which are still largely unknown. In this review article we will highlight the current main topics of our research on genetically caused epilepsies, their pathomechanisms and therapeutic options.
\end{abstract}

Keywords: epilepsy; ion channels; genetics, neuronal excitability

\section{Epilepsie - Zahlen und Ursachen}

Epilepsie betrifft 0.5 bis $1 \%$ der Weltbevölkerung und zählt somit zu den häufigsten neurologischen Erkrankungen. Allein in Deutschland sind rund 600.000 Menschen betroffen, wobei jährlich ca. $30.000 \mathrm{Neu}-$ erkrankungen hinzukommen. Ca. 5\% der Bevölkerung bekommen während ihres Lebens mindestens einen epileptischen Anfall, ohne eine Epilepsie zu entwickeln. Ein epileptischer Anfall entsteht durch eine spontane, unerwünschte elektrische Aktivität von Nervenzellen im Gehirn, die sich in Bewusstseinsstörungen, Muskelzuckungen oder Muskelspannungen bis hin zu komplexen Handlungen äußern können. Treten solche epileptischen Anfälle wiederholt und ohne besonderen Anlass auf, so spricht man von einer Epilepsie. So vielfältig wie das klinische Erscheinungsbild, können auch die in Frage kommenden Ursachen sein. Dabei kann man zwischen symptomatischen Epilepsien, die z.B. durch Tumore, Fehlbildungen, Ischämie oder Enzephalitis verursacht werden können, sowie genetisch bedingten idiopathischen Epilepsien unterscheiden. Bei idiopathischen Epilepsien finden sich keine Hinweise auf eine äußere Ursache oder auf Veränderungen des Gehirns, sie treten häufig altersgebunden auf und machen bis zu $50 \%$ aller Epilepsien aus. Dabei handelt es sich meist um generalisierte Epilepsien, bei denen große Teile des Gehirns in beiden Gehirnhälften betroffen sind. Aber auch bei fokalen Epilepsien, die jeweils nur in einer bestimmten Region in einer Hirnhälfte auftreten (z.B. bei symptomatischen Epilepsien am Ort einer Verletzung oder eines Tumors) wurden genetische Veränderungen gefunden. Selten sind dabei monogene Vererbungen, bei denen einzelne Genmutationen für den resultierenden Phänotyp verantwortlich sind. In den meisten Fällen liegt eine komplexe genetische Vererbung zugrunde, bei der das Zusammenspiel mehrerer genetischer Faktoren die Epilepsie verursacht. Die häufigsten Formen der idiopathischen Epilepsie sind die kindlichen und juvenilen Absence-Epilepsien (CAE, JAE), die juvenile myoklonische Epilepsie (JME) und die Aufwach-Grand-Mal-Epilepsie (EGMA), die polygen vererbt werden und einem komplexen Vererbungsmodus folgen. Die früh beginnende Absence-Epilepsie (EOAE) und die generalisierte/genetische Epilepsie mit Fieberkrämpfen plus (GEFS+) sind dabei eng verwandt. Die meisten Gendefekte, die bei idiopathischen Epilepsien bisher gefunden wurden, betreffen Ionenkanäle, die für die elektrische Weiterleitung im Gehirn notwendig sind.

In der Abteilung Neurologie mit Schwerpunkt Epileptologie der Universität Tübingen werden genau diese Gendefekte untersucht. Ziel dabei ist es, spezifische krankheitsauslösende Gendefekte zu finden, deren molekulare und zelluläre Pathomechanismen $\mathrm{zu}$ verstehen sowie bestehende Therapiemöglichkeiten zu verbessern bzw. neue zu entwickeln. Beispielsweise haben wir in den letzten Jahren mehrere Mutationen im Natriumkanal- 
kodierenden $S C N 2 A$-Gen (Liao et al. 2010a, b), im KCNQ2-Gen, welches die spannungsabhängigen $\mathrm{K}_{\mathrm{V}} 7.2$ - Kaliumkanäle kodiert (Übersicht in Maljevic et al. 2008; Maljevic et al. 2011) sowie in den neuen Epilepsie-Genen SLC2A1 (GLUT1 - Glucosetransporter 1; (Weber et al. 2008)) und PRRT2, das für ein präsynaptisches Protein kodiert (Schubert et al. 2012), beschrieben. Zusammen mit Kooperationspartnern in ganz Europa werden Epilepsiepatienten in Deutschland und im europäischen Ausland rekrutiert und auf genetische Defekte, die zu einer Epilepsie führen können, untersucht. Durch neue Sequenziertechniken, die uns heutzutage zur Verfügung stehen, besteht nun auch die Möglichkeit, exomund genomweit genetische Veränderungen aufzudecken. Genetische Untersuchungen bei idiopathischen Epilepsien sind daher sinnvoll, da sie zusammen mit funktionellen Untersuchungen wissenschaftlich $\mathrm{zu}$ einem besseren Verständnis der Erkrankungen führen, und klinisch weitere unnötige Diagnostik vermeiden, genetische und prognostische Beratung ermöglichen und in manchen Fällen hilfreich für die Therapieentscheidung sein können.

\section{Wie Ionenkanaldefekte zur gestörten neuronalen Erregbarkeit beitragen können}

Die meisten Gendefekte, die bei idiopathischen Epilepsien bisher gefunden wurden, betreffen Ionenkanäle, die für die Erregbarkeit von Nervenzellen verantwortlich sind. Ionenkanäle sind Proteine, die in die Zellmembran von Nervenzellen eingelagert sind und selektive Poren für verschiedene Ionen darstellen (Lerche et al. 2005). Sie können durch Öffnen und Schließen, das sogenannte „Gating“, den Ionenfluss über die Zellmembran beeinflussen und bestimmen so die elektrische Erregbarkeit unserer Nervenzellen im Gehirn. Tragen solche Ionenkanäle eine genetische Veränderung, kann die Funktion des Kanals verändert sein und so wiederum zu einer Änderung der elektrischen Impulse führen. Dies kann die neuronale Erregbarkeit beeinflussen, möglicherweise ein Netzwerk von Neuronen zu einer synchronen Aktivität treiben und so zu einem epileptischen Anfall führen.

Im gesunden Gehirn sorgen Ionenkanäle in den Membranen von erregenden und hemmenden Neuronen - sehr vereinfacht gesehen - für eine neuronale Balance (Abbildung 1). Kommt es zu einer Störung dieser Balance durch Veränderung der Ionenkanalfunktion, kann ein epileptischer

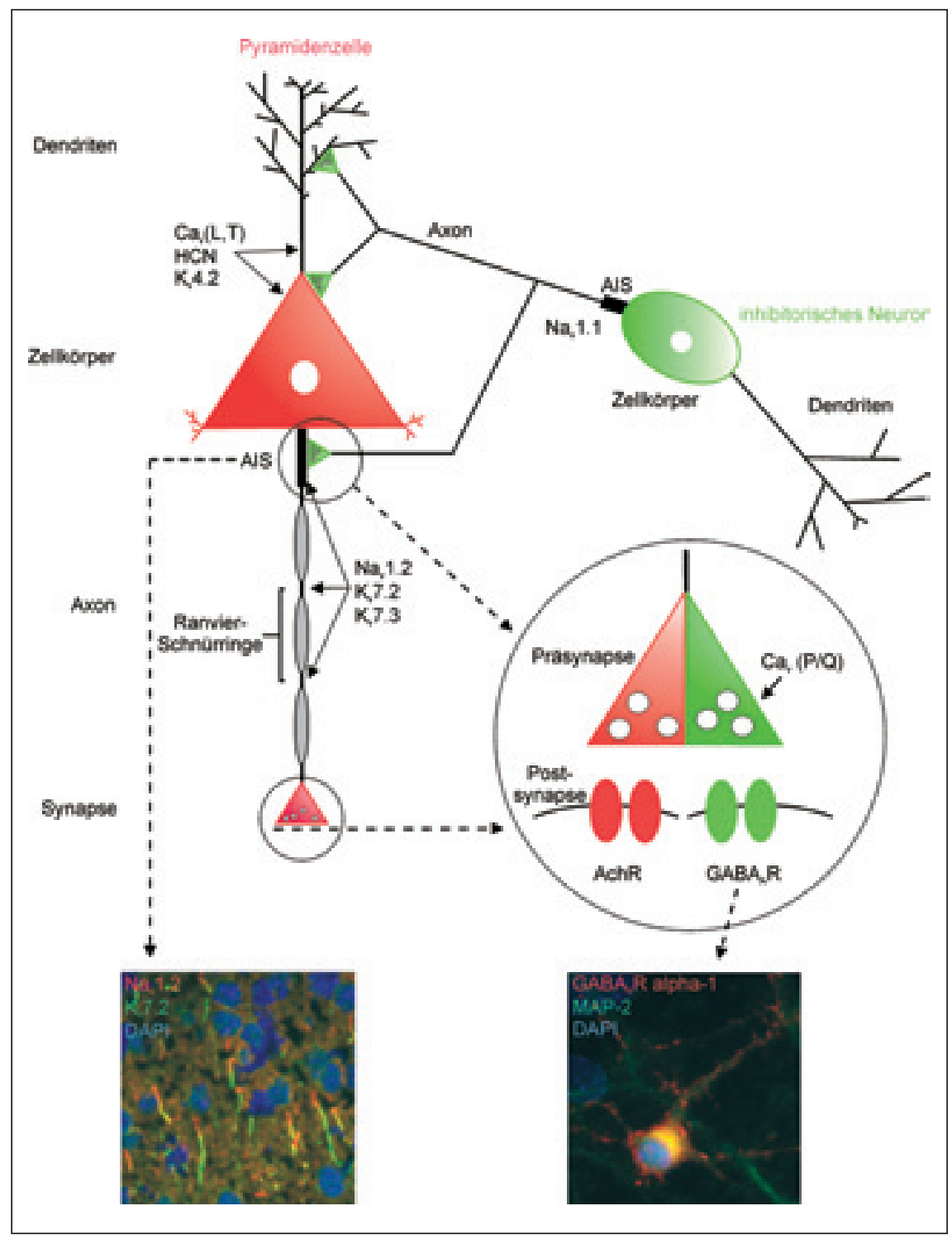

Abb. 1: Lokalisation einiger wichtiger spannungs- und ligandengesteuerter lonenkanäle in erregenden und inhibitorischen Neuronen. Schema einer exzitatorischen Pyramidenzelle (rot) und eines inhibitorischen Neurons (grün) sowie deren synaptische Verbindungen. In den verschiedenen Bereichen der Zellen sind unterschiedliche Typen von Ionenkanälen lokalisiert. Die hier in diesem Artikel beschriebenen sind die somatodendritischen Kalziumkanäle ( $\mathrm{Ca}_{\mathrm{v}}$, T-Typ), $\mathrm{K}_{\mathrm{v}}$ 7.2/7.3 und $\mathrm{Na}_{\mathrm{v}}$ 1.2 im Axoninitialsegment (AIS) und den Ranvierschen Schnürringen von exzitatorischen Neuronen sowie $\mathrm{Na}_{\mathrm{v}} 1.1$ im AIS von inhibitorischen Neuronen. In den präsynaptischen Terminalen finden sich spannungsabhängige Kalziumionenkanäle $\left(\mathrm{Ca}_{\mathrm{v}}, \mathrm{P} / \mathrm{Q}-\mathrm{Typ}\right)$, in den postsynaptischen Membranen ligandengesteuerte $\mathrm{GABA}_{\mathrm{A}}$ - oder Acetylcholinrezeptoren. Links unten: Die immunohistochemischen Färbungen im Hirnschnitt einer adulten Maus zeigen die Kolokalisation von $\mathrm{K}_{\mathrm{v}} 7.2$ (grün)- und $\mathrm{Na}_{\mathrm{v}} 1.2$ (rot)-lonenkanälen am Axoninitialsegment von kortikalen Neuronen. Mit DAPI (blau) wurden die Nuklei der Zellen markiert. Rechts unten: GABA $_{A}$-Rezeptoren (rot, Anti-GABA ${ }_{A}$-alpha1-Untereinheit) in primären hippokampalen Neuronen. Anti-MAP2 -Antikörper (grün) wurde als somatodendritischer Marker verwendet, DAPI (blau) als Nukleus-Marker.

Anfall entstehen. Dies wird auch durch die Tatsache unterstützt, dass die meisten Antiepileptika im klinischen Gebrauch verschiedene Ionenkanaltypen beeinflussen. Bei diesen unterschiedlichen Ionen- kanaltypen handelt es sich um Kanäle für unterschiedliche Ionen, die innerhalb und außerhalb der Zellen vorliegen. Der Ionentransport erfolgt dabei passiv entlang eines elektrochemischen Gradienten, der sich aus 


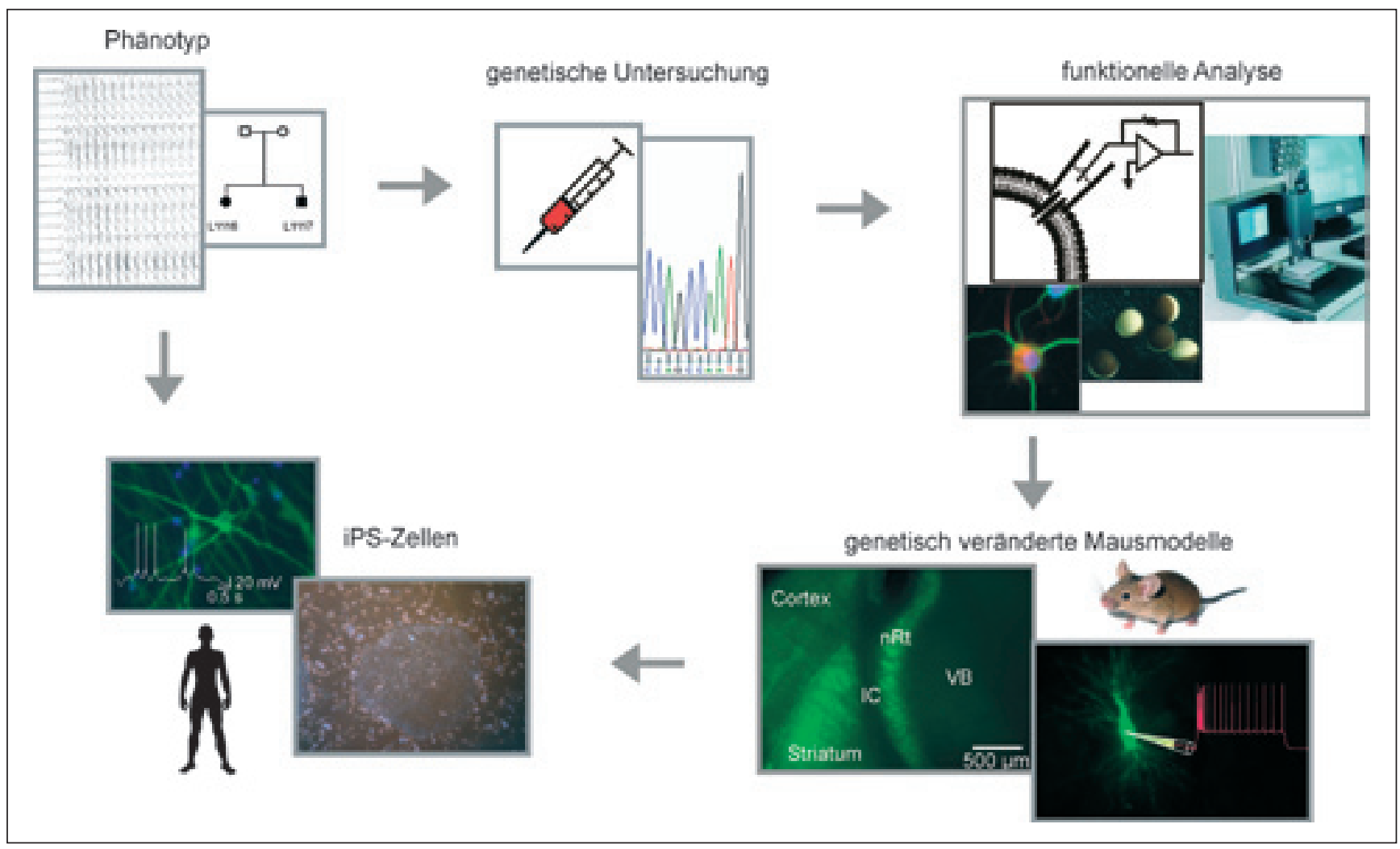

Abb. 2: Heterologe Expressionssysteme, Mausmodelle und iPS-Zellen dienen zur funktionellen Analyse von lonenkanaldefekten. Schematische Darstellung der in unserer Arbeitsgruppe verwendeten Methoden, um die Ursachen idiopathischer Epilepsien aufzuklären. Patienten mit idiopathischen Epilepsien werden genetisch untersucht und mögliche lonenkanaldefekte anschließend in heterologen Expressionssystemen funktionell analysiert. Zur Charakterisierung in einem physiologischeren Hintergrund werden Mausmodelle oder induzierte Pluripotente Stammzellen (iPS-Zellen) von Patienten verwendet.

dem chemischen Konzentrationsgradienten sowie der Potenzialdifferenz (elektrische Triebkraft) zusammensetzt. Die Ionenkanäle, die in unserer Arbeitsgruppe hauptsächlich untersucht werden, sind spannungsgesteuerte $\mathrm{Na}^{+}$- und $\mathrm{K}^{+}$-Kanäle, sowie $\gamma$-Aminobuttersäure $\left(\mathrm{GABA}_{\mathrm{A}}\right)$-Rezeptoren, die durch Bindung von GABA einen Anionenstrom initiieren. Dabei bestimmen die spannungsgesteuerten Ionenkanäle das Ruhemembranpotenzial und sorgen für die Entstehung von Aktionspotenzialen, die Erregungsweiterleitung entlang der Axone sowie für eine Repolarisation der Membran zurück zum Ruhemembranpotenzial. An der präsynaptischen Membran sorgen ankommende Aktionspotenziale für den Einstrom von Kalziumionen in die Zelle und so zur Ausschüttung von chemischen Botenstoffen. Diese sogenannten Neurotransmitter sorgen wiederum für die Weiterleitung der Information zwischen den Zellen. Spezifische spannungsgesteuerte Ionenkanäle in den Axonintialsegemten, den Entstehungsorten der Aktionspotenziale, erregender Pyramidenzellen sind zum Beispiel die $\mathrm{Na}_{\mathrm{v}} 1.2$ - und $\mathrm{Na}_{\mathrm{v}} 1.6-\mathrm{Na}-$ triumionenkanäle, (Liao et al. 2010b) sowie $\mathrm{K}_{\mathrm{V}}$ 7.2- und $\mathrm{K}_{\mathrm{V}}$ 7.3-Kaliumkanäle (Maljevic et al. 2008). In den Axonintialsegmenten der inhibitorischen Neurone dagegen findet sich verstärkt ein weiterer Natriumkanal: $\mathrm{Na}_{\mathrm{v}} 1.1$ (Ogiwara et al. 2007). Spannungsgesteuerte Ionenkanäle können sich in drei verschiedenen Hauptkonformationszuständen befinden, die sich durch Mutationen in den verschiedenen Kanälen verändern können: (i) geschlossen aktivierbar, (ii) offen aktiviert sowie (iii) inaktiviert. Durch eine Mutation innerhalb des Ionenkanalproteins kann es z.B. zu einer Verschiebung der spannungsabhängigen Aktivierung und so zu einem veränderten Ionenfluss kommen. Des Weiteren kann man erregende und hemmende Nervenzellen anhand ihrer chemischen Botenstoffe unterscheiden, die sie für die Erregungsweiterleitung zwischen den Zellen verwenden. Während von erregenden Nervenzellen die Neurotransmitter Glutamat oder Acetylcholin ausgeschüttet werden, findet man bei inhibitorischen Neuronen hauptsächlich den Botenstoff $\gamma$-Aminobuttersäure (GABA) (Abbildung 1). Mutationen an den postsynaptischen
Rezeptoren dieser Botenstoffe können dabei störend auf die Erregungsweiterleitung zwischen den Zellen wirken und so Ursache einer Epilepsie sein. Im Folgenden fassen wir Methoden und Forschungsergebnisse zusammen, welche bisher eine entscheidende Rolle bei der funktionellen Untersuchung von Ionenkanaldefekten gespielt und zur Aufklärung und Therapieerfolgen idiopathischer Epilepsien beigetragen haben.

\section{Funktionelle Untersuchung von Ionenkanaldefekten}

Um die Folgen eines Gendefekts auf die Eigenschaften eines Ionenkanals zu untersuchen, nutzen wir sowohl heterologe Expressionssysteme als auch Tiermodelle. Der erste experimentelle Schritt zum Verständnis der molekularen Physiologie eines Ionenkanals, sowie die oft subtilen Effekte einer genetischen Mutation auf die Funktion des Kanals, ist diese in einem System ohne endogene Kanäle mit der gleichen oder einer ähnlichen Funktion zu exprimieren. Am häufigsten verwendet 
werden dabei unterschiedliche Säugerzelllinien und Xenopus laevis Oozyten. Der Nachteil solcher heterologen Systeme ist die verbleibende Unsicherheit, ob sich die Kanäle wie in ihrer natürlichen neuronalen Umgebung verhalten. Bisher konnte aber für viele Kanäle ein sehr ähnliches Schaltverhalten in heterologen Systemen und natürlichem Gewebe nachgewiesen werden. Dies bestätigt heterologe Expressionssysteme als sehr effiziente erste Untersuchungsmöglichkeit um die resultierenden Ergebnisse und erstellten Hypothesen später in Neuronen zu beweisen. Wir verwenden deshalb dieses System als Bestätigung genetischer Daten und um zu untersuchen, ob eine Änderung des Schaltverhaltens eines defekten Ionenkanals in die Pathogenese der vorliegenden Epilepsie involviert ist. Des Weiteren ermöglichen die in dieser Form relativ rasch untersuchten Ionenkanaldefekte, die zum Beispiel ein neu entdecktes Kandidatengen betreffen oder eine sehr ausgeprägte Veränderung des Schaltverhaltens verursachen, eine Mutation für weitere komplexe Analysen in Tiermodellen und neuronalen Zellen auszuwählen (Abbildung 2). In den letzten Jahren haben wir viele verschiedene Mutationen (z.B. SCN2A-, SCN1A-, $K C N Q 2 / 3$-Mutationen) in heterologen Expressionssystemen untersucht, was im Folgenden kurz zusammengefasst wird. Defekte in spannungsgesteuerten Natriumund Kalium-Ionenkanälen. Mutationen im SCN1A-Gen, dem Gen, das den spannungsgesteuerten $\mathrm{Na}_{\mathrm{v}} 1.1$-Natriumionenkanal kodiert, sind die häufigsten Gendefekte bei idiopatischen Epilepsien. Sie sind mit einem breiten Spektrum der Syndrome assoziiert, die von einer schweren frühkindlichen Enzephalopathie (Dravet-Syndrom) bis hin zur generalisierten/genetischen Epilepsie mit Fieberkrämpfen plus (GEFS+) reichen. Die meisten der Dravet-SyndromPatienten tragen de novo Mutationen, die ein frühes Stopkodon in das SCN1A-Gen einfügen, was in verkürzten Proteinen ohne Funktion resultiert. Interessanterweise verschlechtert die Anwendung des $\mathrm{Na}^{+}$-Ionenkanal-Blockers Lamotrigin die klinische Situation in Dravet-SyndromPatienten. Des Weiteren wurde auch ein Funktionsverlust des $\mathrm{Na}_{\mathrm{v}} 1.1-\mathrm{K}$ anals bei den nicht trunkierenden SCN1A-Mutationen in heterologen Sytemen beschrieben. Da die spannungsabhängigen $\mathrm{Na}^{+}$-Kanäle für die Erzeugung und Weiterleitung von Aktionspotenzialen verantwortlich sind, sollte ein Funktionsverlust („loss-of-function“) dieses Kanals, die Membranerregbarkeit verringern. Wenn dieser Funktionsverlust jedoch hemmende Neurone betrifft, so kann dies zu einer Übererregbarkeit in neuronalen Schaltkreisen führen und damit epileptische Anfälle induzieren.

Benigne familiäre neonatale infantile Anfälle (BFNIS) treten in den ersten Wochen bis Monaten des Lebens auf und werden durch Mutationen im SCN2A-Gen, welches den Natriumionenkanal $\mathrm{Na}_{\mathrm{v}} 1.2$ kodiert, verursacht (Berkovic et al. 2004; Liao et al. 2010a; Liao et al. 2010b). Dabei handelt es sich um sogenannte, gain-offunction“ Mutationen (Abbildung 3), die eine erhöhte Erregbarkeit von Membranen am Axoninitialsegment, dem Ort der Aktionspotenzialentstehung, vorhersagen.

Mutationen im $K C N Q 2$ - und $K C N Q 3$ Gen, den Genen, welche die spannungsabhängigen Kaliumkanäle $\mathrm{K}_{\mathrm{V}} 7.2$ und $\mathrm{K}_{\mathrm{V}} 7.3$ kodieren, führen $\mathrm{zu}$ einer Epilepsie des Neugeborenenalters (Benigne familiäre Neugeborenenkrämpfe; BFNS) mit An-

\section{Incredible discoveries with Fine Science Tools}




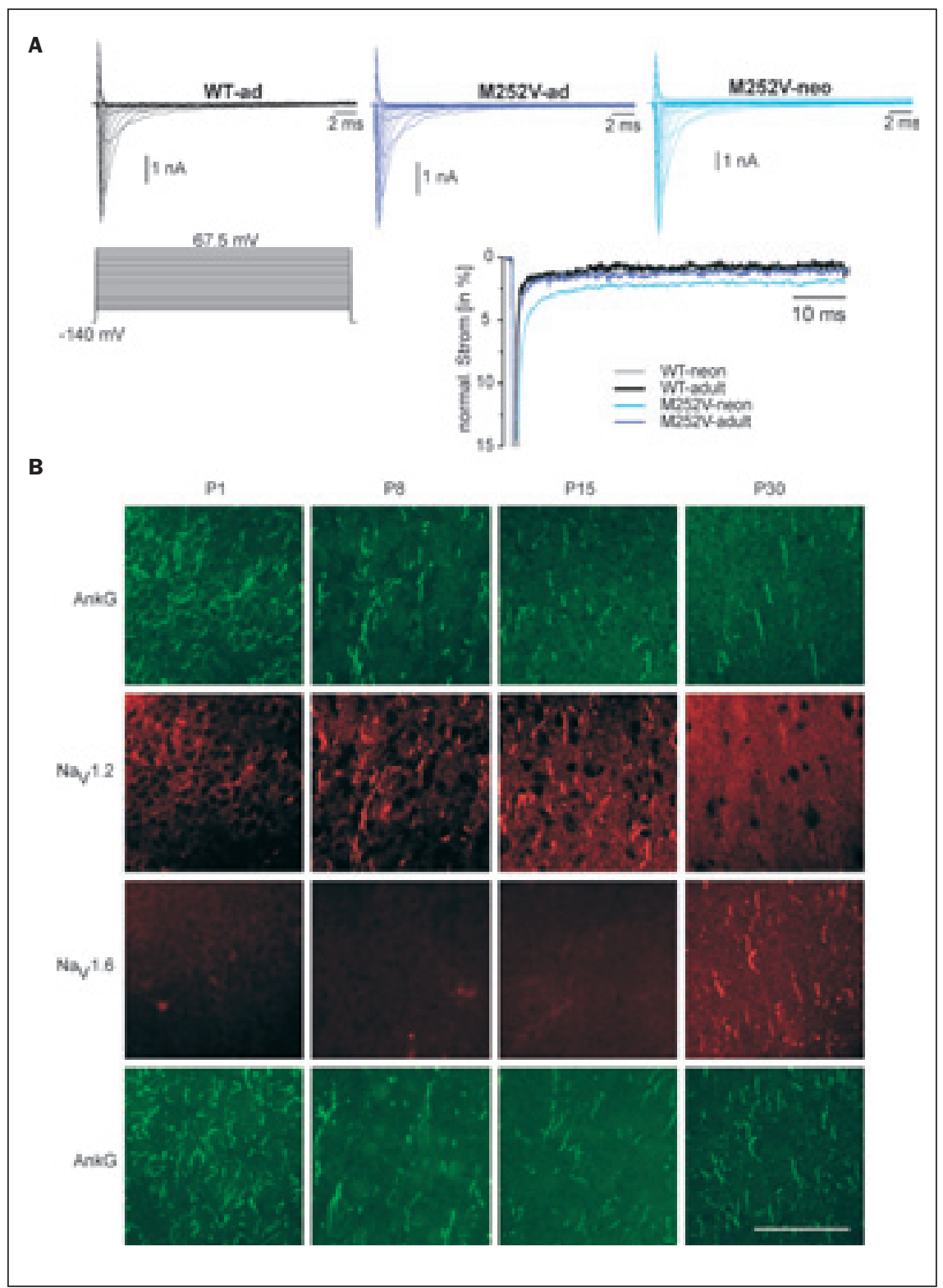

Abb. 3: SCN2A-Mutationen führen zur Steigerung der Kanalfunktion. A) Eine mit BFNIS assozilerte Mutation wurde in die neonatale und adulte Splicevariante des $\mathrm{Na}_{\mathrm{v}}$ 1.2.-Kanals eingebaut und in tSA201-Zellen analysiert (Liao et al. 2010b). Diese Mutation zeigte einen Gain-of-function, in dem sie die Inaktivierung dieses Kanals beeinflusst und den sogenannten persistierenden Strom erhöht (siehe normalisierten Strom unten rechts), was zu einer Depolarisation der Zelle führt und so die Erregbarkeit erhöht. Interessanteweise ist dieser Effekt nur bei der neonatalen Splicevariante zu sehen, was die Altersabhängigkeit dieses Epilepsiesyndroms erklären könnte. Dennoch werden bei anderen SCN2A-Mutationen solche Effekte entweder bei beiden oder sogar bei der adulten Variante gefunden. B) Um die Altersabhängigkeit besser zu verstehen, wurden kortikale Hirnschnitte von Mäusen unterschiedlichen Alters mit spezifischen Antikörpern gegen $\mathrm{Na}_{\mathrm{v}} 1.2, \mathrm{Na}_{\mathrm{v}} \mathrm{1.6}$ (rot), Ankyring als Axoninitialsegmentmarker (AnkG-grün) und DAPI (blau) gefärbt. In den frühen Stadien ist hauptsächlich der $\mathrm{Na}_{\mathrm{v}} \mathbf{1 . 2}$ Kanal in den Axoninitialsegmenten von kortikalen Neuronen zu erkennen, welcher später vom $\mathrm{Na}_{\mathrm{v}}$ 1.6-Ionenkanal ersetzt wird. Maßstabsbalken $50 \mu \mathrm{m}$. Modifiziert nach Liao et al. 2010b.

fällen, die in den ersten Lebenstagen auftreten und spontan nach wenigen Monaten sistieren. In selteneren Fällen kommt es zu weitaus schwereren Verlaufsformen, u.a. mit der Entwicklung von Enzephalopathien (Weckhuysen et al. 2012). In Ausnahmefällen kommt es darüber hinaus auch zu einer Beteiligung des peripheren Nervensystems im Sinne einer Hyperexzitabilität (Myokymie, Neuromyotonie), die entweder isoliert oder in Kombination mit epileptischen Anfällen auftreten kann (Übersicht: Maljevic et al. 2008). Pathophysiologische Studien in heterologen Expressionssystemen zeigten, dass alle KCNQ2-Mutationen zu einem Funktionsverlust des Kanals führen. Als molekulare Mechanismen wurden veränderte Proteinstabilität, intrazellulärer Transport oder Veränderungen des Schaltverhaltens genannt (Maljevic et al. 2008). Eine Charakterisierung einer solchen KCNQ2-Mutation, die BFNS in einer türkischen Familie verursacht, ist in Abbildung 4 gezeigt (Maljevic et al. 2011).

Dem aktuellen Kenntnisstand nach spielen die $K_{v}$ 7.2- und $K_{v}$ 7.3-Kanäle eine Schlüsselrolle bei der Regulierung des Membranpotenzials im Subschwellenbereich von Aktionspotenzialen. Eine reduzierte Aktivität dieser Kanäle führt hierbei zu einer leichten depolarisierenden Verschiebung des Membranpotenzials in diesem Bereich, was in einem schnelleren und häufigeren Überschreiten der Aktionspotenzialschwelle und somit in einer verstärkten Erregbarkeit resultiert. Ein umgekehrter Mechanismus, welcher das Membranpotenzial in diesem Bereich in die entgegengesetzte Richtung verschiebt, könnte für die Behandlung von Epilepsien, aber auch anderer Erkrankungen, die mit einer erhöhten neuronalen Erregbarkeit einhergehen, bedeutsam sein. $\mathrm{K}_{\mathrm{V}} 7-\mathrm{Ka}$ näle stellen deshalb eine sehr interessante pharmakologische Zielstruktur dar, deren Effektivität bereits anhand des neuen Antiepileptikums Retigabin offenbar wurde (Orhan et al. 2012; Wuttke et al. 2005).

Defekte in ligandgesteuerten Ionenkanälen: GABA $A_{A}$ Rezeptoren. GABA $\mathrm{A}_{\mathrm{A}}$-Rezeptoren sind die wichtigsten inhibitorischen Rezeptoren im Zentralnervensystem (ZNS). Sie werden aus fünf verschiedenen Untereinheiten zusammengesetzt. Insgesamt existieren 17 unterschiedliche Untereinheiten, die in acht Klassen ( $\alpha$ bis $\rho)$ eingeteilt werden. Die meisten GABA - $^{-}$ Rezeptoren im ZNS bestehen aus zwei $\alpha 1$-, zwei $\beta 2$ - und einer $\gamma 2$-Untereinheit. Verschiedene GABA $_{A}$-Rezeptor-Mutationen, die zur Entstehung von Epilepsien beitragen, wurden bereits beschrieben (Weber und Lerche 2008). Die erste Mutation in der $\gamma 2$-Untereinheit wurde in Familien mit kindlicher Absence Epilepsie (CAE) und generalisierten Epilepsien mit Fieberkrämpfen plus (GEFS+) gefunden und die meisten bis heute entdeckten Mutationen befinden sich in dieser Untereinheit. Weitere Mutationen wurden in den $\alpha 1$-, 
$\alpha 6$ - und $\delta 6$-Untereinheiten gefunden (Galanopoulou 2010; Hernandez et al. 2011). Alle bisher beschriebenen Mutationen zeigten in heterologen Expressionssystemen wie erwartet einen Funktionsverlust. Bei der a1-S326fs328X-Mutation beispielsweise zeigte sich diese durch eine geringere Stabilität des Proteins, das dadurch nicht in die Plasmamembran der Zellen eingebaut wird (Maljevic et al. 2006).

Wo und wann werden die verschiedenen Kanäle exprimiert? Obwohl die oben genannten funktionellen Untersuchungen zu einem besseren Verständnis der zugrunde liegenden Krankheit führen, bleiben viele Fragen ungeklärt. Um die funktionellen Krankheitsmechanismen besser verstehen zu können, sind physiologische Systeme notwendig, die natürlichere Bedingungen widerspiegeln (Abbildung 2). Dafür eignen sich beispielsweise Nervenzellen aus Wildtyp- oder genetisch veränderten Mauslinien. Um die Funktion von Ionenkanaldefekten im neuronalen Hintergrund untersuchen zu können, können mutierte Ionenkanäle in primäre murine Neuronen- kulturen über Transfektion, Elektroporation oder virale Infektion eingebracht werden. Da primäre Neurone elektrisch erregbar sind und somit viele endogene Ionenkanäle - auch die zu untersuchenden - exprimieren, ist eine funktionelle Analyse spezifischer in die Zellen eingebrachter Kanäle allerdings oft nicht einfach. Spezifische Knock-out-Mäuse, bei denen ein bestimmtes Gen außer Gefecht gesetzt und somit die Expression des kodierten Proteins unterbunden wird, können für die funktionelle Analyse eines spezifischen Ionenkanaldefekts genutzt werden. So können WT oder mutierte Ionenkanäle in primären Knock-out-Zellen exprimiert und Feuerungsverhalten sowie veränderte Ionenströme von Neuronen mit induziertem Ionenkanaldefekt untersucht werden und so Einblicke in den Krankheitsmechanismus geben. In den letzten Jahren wurde auch häufiger die in utero Elektroporation verwendet, um spezifische neuronale Vorläuferzellen in bestimmten Gehirnregionen genetisch zu manipulieren. Die Transfektion von neuronalen Vor- läuferzellen einer bestimmten Gehirnregion wird dabei durch Mikroinjektion von Plasmid-DNA in das Gehirn embryonaler Mäuse im Uterus des Muttertieres und anschließender elektrischer Stimulation des Gehirns durchgeführt. 70-90\% der geborenen Jungtiere exprimieren bei dieser Methode das transfizierte Plasmid. Durch Co-Expression von fluoreszierendem Protein können die transfizierten Zellen auch während ihrer Entwicklung untersucht werden.

Wie oben bereits beschrieben sind spannungs- und ligandenabhängige Ionenkanäle an unterschiedlichen Bereichen verschiedener Nervenzellen lokalisiert (Abbildung 1). Für die Untersuchung der zellulären Lokalisation bestimmter Ionenkanäle in der Zelle und in verschiedenen Gehirnbereichen werden primäre neuronale Zellen aus Hippocampi von Mäuseembryonen kultiviert oder murine Hirnschnitte verwendet und beispielsweise mittels immunohistochemischer Methoden angefärbt. Viele idiopathische Epilepsiesyndrome weisen eine charakteristische
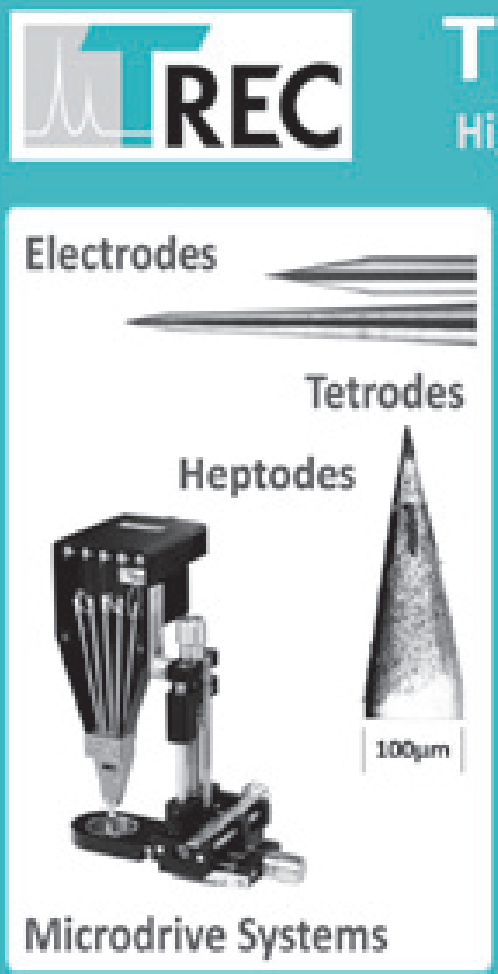

\section{Thomas RECORDING GmbH High Tech Made in GERMANY - info@ThomasRECORDING.com}

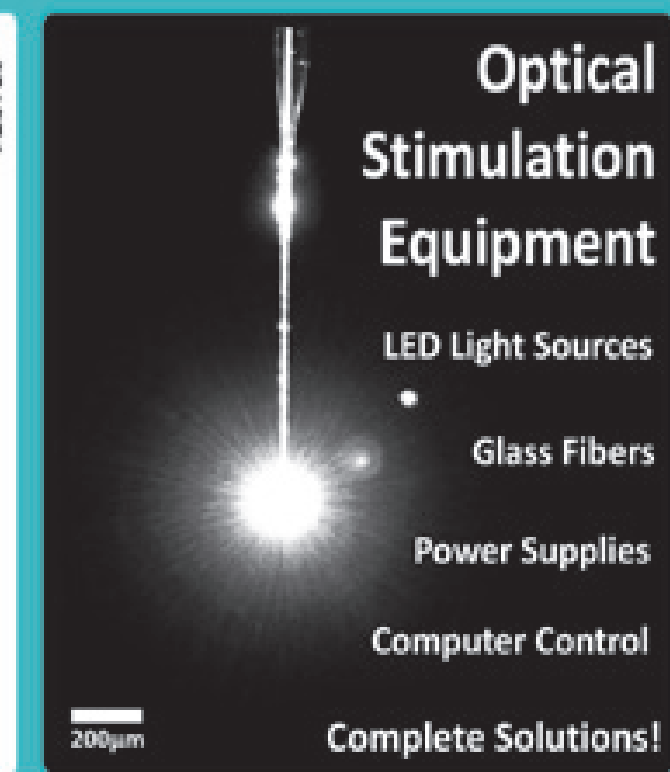

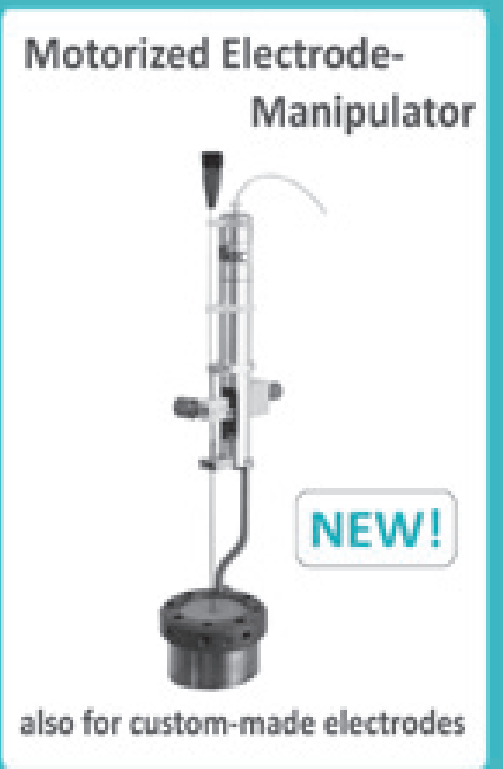


Altersabhängigkeit auf, bei denen der molekulare Mechanismus meist ungeklärt ist. Mithilfe von Antikörperfärbungen kann eine altersabhängige Expression verschiedener Ionenkanäle untersucht werden (Abbildung 3). So konnte in Hirnschnitten verschiedener Entwicklungsstufen der Maus gezeigt werden, dass in den frühen Stadien hauptsächlich der Natriumionenkanal $\mathrm{Na}_{\mathrm{v}} 1.2$ in den Axoninitialsegmenten von hippokampalen und kortikalen Pyramidenzellen exprimiert und später vom spannungsabhängigen $\mathrm{Na}_{\mathrm{v}}$ 1.6-Ionenkanal ersetzt wird (Liao et al. 2010b). Dieser Wechsel in der Expression der beiden $\mathrm{Na}-$ triumionenkanäle kann eine Erklärung für die altersabhängigen Anfälle bei BFNIS sein, die durch Mutationen im SCN2A-Gen hervorgerufen werden.

\section{Genetisch veränderte Mausmodelle tragen zum Verständnis von Epilepsiemechanismen bei}

Um die Auswirkungen einer bei Epilepsiepatienten gefundenen Mutation auf neuronaler Ebene und so in physiologischeren Systemen zu untersuchen, verwenden wir zusätzlich genetisch veränderte Mausmodelle, die eine menschliche, zu einer bestimmten Epilepsie führende Mutation tragen. Durch die Nutzung eines solchen „humanisierten Mausmodells“ können wir die Krankheitsmechanismen in einzelnen Nervenzellen, in neuronalen Netzwerken sowie im lebenden Organismus untersuchen. Transgene sowie spontan mutierte Mäuse haben sich als wichtiges Werkzeug bei der Identifikation neuer molekularer
Mechanismen von Epilepsie bewährt, können mutationstragende Kandidatengene, die bei Epilepsiepatienten und deren Familien gefunden wurden, als Verursacher bestätigen, sowie zur Klärung der pathogenenen Mechanismen und Therapiemöglichkeiten beitragen. So konnten beispielsweise pathogene Mechanismen bei Ionenkanaldefekten in exzitatorischen und inhibitorischen Neuronen aufgeklärt werden, die zuvor unbekannt waren. Wie bereits oben beschrieben, gibt es verschiedene Typen von Natriumkanälen, die in exzitatorischen und inhibitorischen GABAergen Neuronen exprimiert werden und in unterschiedlicher Weise zur Exzitabilität der Zellen beitragen. Knock-out- und Knock-in-Mausmodelle zeigten, dass SCN1A-Deletionen zu vermindertem Feuern inhibitorischer Neurone

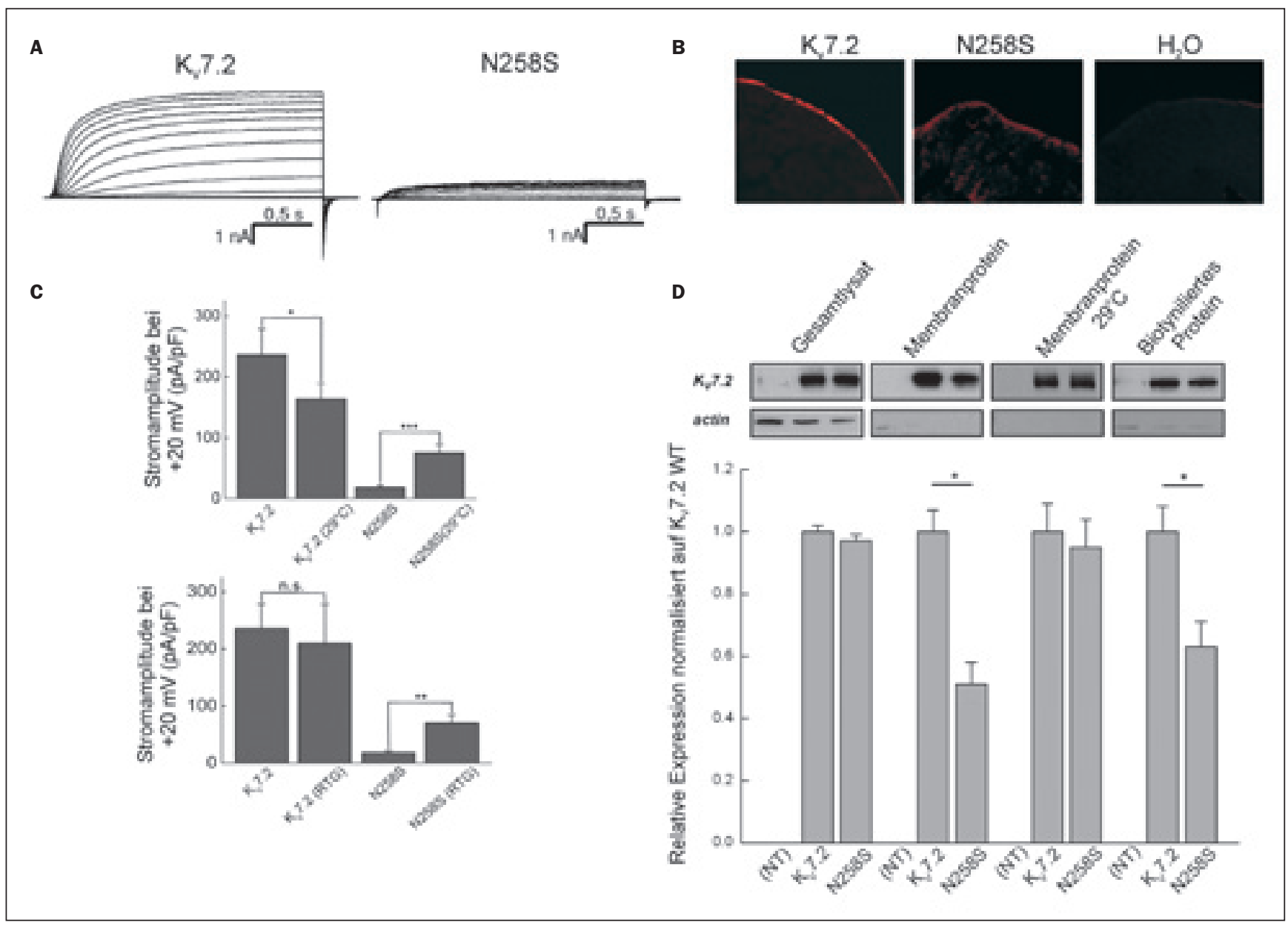

Abb. 4: Charakterisierung der $\mathrm{K}_{\mathrm{v}}$ 7.2-N258S-Mutation. Diese Mutation wurde in Xenopus laevis Oozyten und in CHO-Zellen (chinesische Hamster-Oozyten) untersucht und ein Defekt des intrazellulären Transports (sogenanntes „trafficking“) festgestellt. A) Die repräsentative Stromdichte ist für die N258S-Mutation in CHO-Zellen stark reduziert. B) Färbungen in Xenopus laevis Oozyten ergeben eine spezifische Membranfärbung für $K_{v} 7.2$ WT-Kanäle, nicht jedoch für N258S-Kanäle. C) Durch erniedrigte Temperatur bei der Kultivierung der Zellen sowie Applikation des Pharmakons Retigabin, das an diese Kanäle bindet, konnten die Folgen des Defekts deutlich vermindert werden, was durch die Steigerung der maximalen Stromdichten bei den N258S-Kanälen zu sehen ist. Beide Prozesse führen vermutlich zu einer verbesserten Proteinfaltung und können so den Transport zur und die Expression in der Zellmembran verbessern. D) Diese Befunde wurden mittels einer biochemischen Analyse bestätigt. Modifiziert nach Maljevic et al. 2011. 


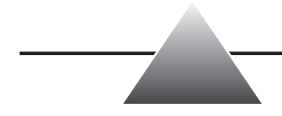

und wahrscheinlich so zu Anfällen bei Dravet-Syndrom- und GEFS+-Patienten führen (Ogiwara et al. 2007; Yu et al. 2006). Eine der ersten beschriebenen GEFS+-Mutationen, die im SCN1A-Gen gefunden wurde, ist die R1648H-Mutation im Spannungssensor des $\mathrm{Na}_{\mathrm{v}} 1.1-$ Kanals (Escayg et al. 2000). Durch die Untersuchung des Knock-in-Mausmodells mit dieser Mutation (Martin et al. 2010) konnten wir zeigen, dass inhibitorische Neurone in verschiedenen Hirnregionen eine deutliche Reduktion in ihrer Feuerfrequenz zeigen (Hedrich et al. 2012). Eine verminderte Inhibition in Kortex, Thalamus und Hippocampus führt vermutlich zu epileptischen Anfällen bei den Tieren und auch bei den betroffenen Patienten. Aber nicht nur ein Defekt in der $\alpha$-Untereinheit des $\mathrm{Na}_{\mathrm{v}} 1.1$-Natriumkanals kann zu GEFS+ führen, es wurden auch Mutationen im SCN1BGen gefunden, welches die $\beta 1$-Unterheit verschiedener $\mathrm{Na}^{+}-\mathrm{Kanäle}$ kodiert. Mithilfe einer Knock-in-Maus konnte gezeigt werden, dass heterozygote Tiere mit der SCN1B-C121W-Mutation eine erhöhte Körpertemperatur und einen ähnlichen Phänotyp aufweisen, wie betroffene Patienten (Wimmer et al. 2010). Die WT $\beta 1$-Unterheit wird hauptsächlich in den Axoninitialsegmenten von Pyramidenzellen exprimiert und fehlt bereits bei heterozygoten Tieren weitgehend. So konnte gezeigt werden, dass die $\beta 1$-Unterheit die Exzitabilität am AIS moduliert und eine Epilepsie auslösen kann, wenn diese Modulation gestört ist. Auch die Krankheitsmechanismen bei BFNIS untersuchen wir derzeit mithilfe eines Knock-in-Mausmodells. So konnten wir in ersten Experimenten mit einer neu generierten Knock-in-Maus mit einer Mutation im SCN2A-Gen (Kooperation mit Dirk Isbrandt, Hamburg) die Ergebnisse von heterologen Expressionssystemen, bei denen es zu einem klaren ,gain-of-function“ kommt, bestätigen. Der Defekt im Na 1.2-Kanal führt dabei zu einer erhöhten Feuerfrequenz hippokampaler Pyramidenzellen und somit zu einer gesteigerten Exzitabilität in heterozygoten Tieren (Engeland et al. 2012).

Obwohl bisher mithilfe von transgenen Mausmodellen grundlegende Erkenntnisse über idiopathische Epilepsien getroffen werden konnten, bleiben doch einige Probleme bestehen. So kann der Krankheitsverlauf innerhalb einer Familie mit demselben Gendefekt oft sehr variabel sein. Die Mechanismen dahinter sind bisher wenig verstanden. Bei verschiedenen Mausmodellen konnte gezeigt werden, dass der genetische Hintergrund der Tiere eine wichtige Rolle bei der Ausprägung des Phänotyps sowie bei der Schwelle eines ausgelösten epileptischen Anfalls spielt. Die Beteiligung von spezifischen Ionenkanaldefekten auf die Exzitabilität des Gehirns ist also sehr komplex, selbst wenn es sich eindeutig um ein betroffenes Gen handelt, sei es in Maus oder Patient (Glasscock et al. 2007; Martin et al. 2007).

\section{Induzierte pluripotente Stammzellen}

Die bisher erwähnten Modelle, die viele Kenntnisse über Ionenkanäle und deren Funktion im Gehirn geliefert haben, weisen jedoch einige Limitationen auf: (i) bei den in vitro verwendeten Zelllinien handelt es sich um keine Neuronen-ähnlichen Zellen und besitzen somit nicht die Eigenschaften der bei Epilepsie primär betroffenen Zellpopulationen; (ii) es kann immer nur eine bekannte Mutation untersucht werden, was es schwierig macht, polygene Pathomechanismen zu erkennen und (iii) die Untersuchungen an Tiermodellen können nur zu einem gewissen Grad auf den Menschen übertragen werden, da die Regulation und die physiologische Rolle eines Proteins unterschiedlich zum menschlichen Organismus sein könnte und Kompensationsmechanismen des Tiermodells Auswirkungen von Mutationen beeinflussen können. Daher ist die Erforschung der Pathogenese an menschlichen, möglichst Neuronen-ähnlichen Zellen,

\section{Electronic Instruments for the Life Sciences}

\section{made to measure}

NEW Low-Price Instrument Series

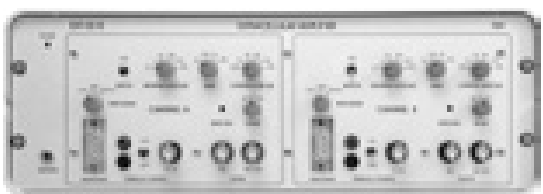

EXT-02 B

Extracellular

amplifier with

filters, gain and

stimulus control

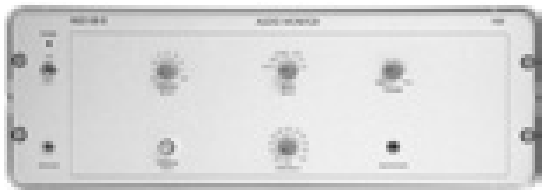

AUD-08 B

Audio monitor for

up to four EXT-02 B

amplifiers

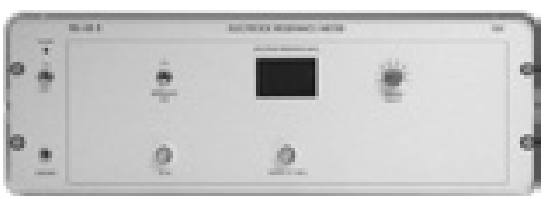

REL-08 B

Resistance test for up to four EXT-02 B

amplifiers

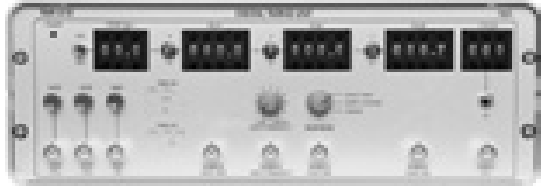

TMR-01B

Versatile timer

with pulse or

burst mode

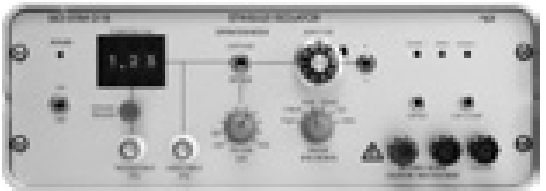

ISO-STIM 01B

Isolated stimulator with voltage or current output and interphase gap

\section{npi provides complete rigs for electrophysiology}

npi is distributing:

- ALA Scientific perfusion systems and accessories

- Burleigh micropositioners and mounts

- Campden vibrating microtomes

- DataWave data acquisition systems

- Lumen Dynamics X-Cite fluorescence illumination

- Molecular Devices amplifiers and data acquisition

- NeuroNexus acute and chronic electrodes

- Scientifica micropositioners, mounts, Slicescope two-photon Slicescope

- Sensapex piezo driven micromanipulator

- TMC vibration isolation tables

\section{npi electronic $\mathrm{GmbH}$}

Phone + 49 (0)7141-97302-30; Fax: +49 (0)7141-97302-40 support@npielectronic.com; http://www.npielectronic.com 
welche zusätzlich den gleichen genetischen Hintergrund haben wie der Ursprung der Mutation, eine interessante Option. Ein solcher Ansatz bietet sich seit Kurzem durch die Reprogrammierung von HautFibroblasten von Patienten in induzierte Pluripotente Stammzellen (iPS-Zellen). Diese „reprogrammierte Zellpopulation“ ist in der Lage, in neuronale, glutamaterge und GABAerge Zellen zu differenzieren (Koch et al. 2009; Takahashi et al. 2007), die weiter biochemisch, morphologisch und elektrophysiologisch untersucht werden können. Die ausdifferenzierten Zellen von Patienten, die bestimmte Genmutationen tragen, können im Vergleich mit denen von altersgleichen Kontrollpersonen neue Einblicke in die Funktion und die Entstehung von idiopathischen Epilepsien anbieten. Weiterhin können diese Zellen für Medikamenten-Screening verwendet werden und als Einsatz für die individuelle Therapie weiterentwickelt werden. Wir arbeiten derzeit an der Entwicklung solcher humanen Modelle von Patienten mit genetischer Epilepsie und hoffen, unser Verständnis dieser Krankheit weiter zu vertiefen.

\section{Literatur}

Liao, Y., Deprez, L., Maljevic, S., Pitsch, J., Claes, L., Hristova, D., Jordanova, A., AlaMello, S., Bellan-Koch, A., Blazevic, D., et al. (2010b): Molecular correlates of age-dependent seizures in an inherited neonatal-infantile epilepsy. Brain: A journal of neurology 133: 1403-1414.

Maljevic, S., Krampfl, K., Cobilanschi, J., Tilgen, N., Beyer, S., Weber, Y.G., Schlesinger, F., Ursu, D., Melzer, W., Cossette, P., et al. (2006): A mutation in the GABA(A) receptor alpha(1)-subunit is associated with absence epilepsy. Ann Neurol 59: 983-987.

Maljevic, S., Naros, G., Yalcin, O., Blazevic, D., Loeffler, H., Caglayan, H., Steinlein, O.K., and Lerche, H. (2011): Temperature and pharmacological rescue of a foldingdefective, dominant-negative $\mathrm{K}_{\mathrm{v}} 7.2$ mutation associated with neonatal seizures. Hum Mutat 32: E2283-2293

Weber, Y.G. und Lerche, H. (2008): Genetic mechanisms in idiopathic epilepsies. Dev Med Child Neurol 50: 648-654.

Wimmer, V.C., Reid, C.A., Mitchell, S., Richards, K.L., Scaf, B.B., Leaw, B.T., Hill, E.L., Royeck, M., Horstmann, M.T., Cromer, B.A., et al. (2010): Axon initial segment dysfunction in a mouse model of genetic epilepsy with febrile seizures plus. $J$ Clin Invest 120: 2661-2671.

\section{Kurzbiografien}

Ulrike Hedrich studierte Biologie an der Universität Ulm und promovierte 2008 bei PD Dr. Wolfgang Stein in der Abteilung
Neurobiologie (Leitung Prof. Dr. Harald Wolf) an der Universität Ulm über die Aktivierung und das Zusammenspiel höherer neuronaler Zentren auf zentrale Mustergeneratoren im stomatogastrischen Nervensystem des Taschenkrebses. Danach wechselte sie als Postdoktorandin zu Prof. Dr. Holger Lerche in die Abteilungen Neurologie und Angewandte Physiologie an der Universität Ulm. Seit 2010 ist sie Postdoktorandin am Hertie-Institut für klinische Hirnforschung in der Abteilung Neurologie mit Schwerpunkt Epileptologie unter der Leitung von Prof. Dr. Holger Lerche, wo sie sich insbesondere mit der Untersuchung von Natriumkanaldefekten in Mausmodellen beschäftigt.

Snezana Maljevic studierte Molekularbiologie und Physiologie an der Universität Belgrad, Serbien und promovierte im Jahr 2005 an der Universität Ulm über molekulare Mechanismen erblicher Epilepsien bei Prof. Dr. Holger Lerche. In seiner Arbeitsgruppe, die im Januar 2010 nach Tübingen ins Hertie-Institut für klinische Hirnforschung wechselte, beschäftigt sie sich als Postdoktorandin und Projektleiterin in der Abteilung Neurologie mit Schwerpunkt Epileptologie mit unterschiedlichen Projekten, insbesondere interessiert sie sich für die neuronale Expression von Ionenkanälen und die Mechanismen von Kaliumkanaldefekten.

Holger Lerche studierte Physik und $\mathrm{Hu}-$ manmedizin an der Ludwig-MaximiliansUniversität München und promovierte 1996 über Mechanismen von Myotonien bei Prof. Dr. Lehmann-Horn. Während seiner Tätigkeit als Facharzt in der Neurologischen Klinik der Universität Ulm habilitierte er sich im Jahr 2000 über die Pathophysiologie von Ionenkanalerkrankungen der Muskulatur und des Gehirns. Er war dann als Oberarzt und Leiter der Epileptologie an der Neurologischen Klinik der Universität Ulm tätig und war 2003-2008 Heisenberg-Stipendiat. Gastaufenthalte 2001 in der Klinik für Epileptologie der Universität Bonn, 2005 am Institute of Neurology, Queen Square, London und 2007 an der University of Melbourne. 2009 erhielt er den Ruf auf den Lehrstuhl für Neurologie mit Schwerpunkt Epileptologie an der Medizinischen Fakultät der Universität Tübingen. Seine dortige Arbeitsgruppe befasst sich mit der Genetik und Pathophysiologie erblicher Epilepsiesyndrome und verwandter neurologischer Erkrankungen, der damit zusammenhängenden Erregbarkeit von
Nervenzellen sowie der Funktion, Expression und Pharmakologie von Ionenkanälen und -transportern.

\section{Korrespondenzadressen}

Prof. Dr. med. Holger Lerche Abt. Neurologie mit Schwerpunkt Epileptologie

Hertie-Institut für Klinische Hirnforschung Eberhard-Karls-Universität Tübingen

Hoppe-Seyler-Str. 3, 72076 Tübingen

Tel.: $\quad+4970712980442(-80466)$

Fax: $\quad+497071294488$

E-Mail:holger.lerche@uni-tuebingen.de

\section{Dr. Ulrike Hedrich}

Abt. Neurologie mit Schwerpunkt Epileptologie

Hertie-Institut für Klinische Hirnforschung Eberhard-Karls-Universität Tübingen Otfried-Müller-Straße 27, 72076 Tübingen

Tel.: $\quad+4970712981921$

Fax: $\quad+497071294698$

E-Mail: ulrike.hedrich@uni-tuebingen.de

\section{Dr. Snezana Maljevic}

Abt. Neurologie mit Schwerpunkt Epileptologie

Hertie-Institut für Klinische Hirnforschung Eberhard-Karls-Universität Tübingen

Otfried-Müller-Straße 27, 72076 Tübingen

Tel.: $\quad+4970712981922$

Fax: $\quad+497071294698$

E-Mail:snezana.maljevic@uni-tuebingen.de

(C) Springer-Verlag GmbH 2013 